\title{
Update of Neuromodulation in Chronic Migraine
}

\author{
Hsiangkuo Yuan ${ }^{1}$ (D) Tzu-Ying Chuang ${ }^{2}$ \\ Accepted: 15 September 2021 / Published online: 11 November 2021 \\ (C) The Author(s), under exclusive licence to Springer Science+Business Media, LLC, part of Springer Nature 2021
}

\begin{abstract}
Purpose of Review Neuromodulation devices have become an attractive alternative to traditional pharmacotherapy for migraine, especially for patients intolerant to medication or who prefer non-pharmacological options. In the past decades, many studies demonstrated the efficacy of neuromodulation devices in patients with episodic migraine (EM). However, the benefit of these devices on chronic migraine $(\mathrm{CM})$, which is typically more debilitating and refractory than EM, remains not well studied.

Recent Findings We reviewed the literature within the last five years on using FDA-cleared and investigational devices for CM. There were eight randomized controlled trials and 15 open-label observational studies on ten neuromodulation devices. Summary Neuromodulation is promising for use in CM, although efficacy varies among devices or individuals. Noninvasive devices are usually considered safe with minimal adverse events. However, stimulation protocol and methodology differ between studies. More well-designed studies adhering to the guideline may facilitate FDA clearance and better insurance coverage.
\end{abstract}

Keywords Headache $\cdot$ Neuromodulation $\cdot$ Chronic migraine $\cdot$ Device $\cdot$ Electrical stimulation

\section{Introduction}

Migraine affects more than $10 \%$ of the general population and is one of the most prevalent medical conditions in the world [1]. Approximately 3\% of people with episodic migraine (EM) transform to chronic migraine (CM), defined as 15 or more monthly headache days with eight or more being migrainous, leading to increased disability and reduced quality of life. In the last two decades, noninvasive neuromodulation devices have become an effective alternative to pharmacological treatment for migraine due to a better mechanistic understanding of headache pathophysiology and burgeoning technological advances. Due to its noninvasive nature, peripheral neuromodulation is generally regarded as a safe and convenient option. The United

This article is part of the Topical Collection on Neuromodulation

Hsiangkuo Yuan

hsiangkuo.yuan@jefferson.edu

1 Jefferson Headache Center, Department of Neurology, Thomas Jefferson University Hospital, 900 Walnut St. Suite 200, PA 19107 Philadelphia, USA

2 Department of Neurology, Thomas Jefferson University Hospital, 909 Walnut St., PA 19107 Philadelphia, USA
States Food and Drug Administration (FDA) has cleared five devices for headache treatment (Fig. 1) in the past ten years; only a few are indicated for CM. In this chapter, we review migraine trials explicitly designed for or included $\mathrm{CM}$ in the past five years on FDA-cleared and investigational devices (Table 1, Table 2).

\section{FDA-Cleared Devices}

\section{Vagus Nerve Stimulation}

The gammaCore Sapphire ${ }^{\mathrm{TM}}$ is a handheld noninvasive vagus nerve stimulator (nVNS) developed by electroCore, Inc. (Basking Ridge, NJ). Patients apply the device to the vagus nerve transcutaneously on either side of the neck. The FDA cleared gammaCore Sapphire ${ }^{\mathrm{TM}}$ in April 2017 for the acute treatment of pain associated with episodic cluster headache in adults. Now, it is FDA-cleared for acute and preventative treatment of pain for migraine patients aged 12 years or older.

There are four major nVNS trials for migraine, two for $\mathrm{EM}$, one specifically designed for $\mathrm{CM}$, and another one that included CM. The first nVNS study for CM was a pilot feasibility study (The EVENT study), with primary endpoints being safety/tolerability. This randomized double-blind 


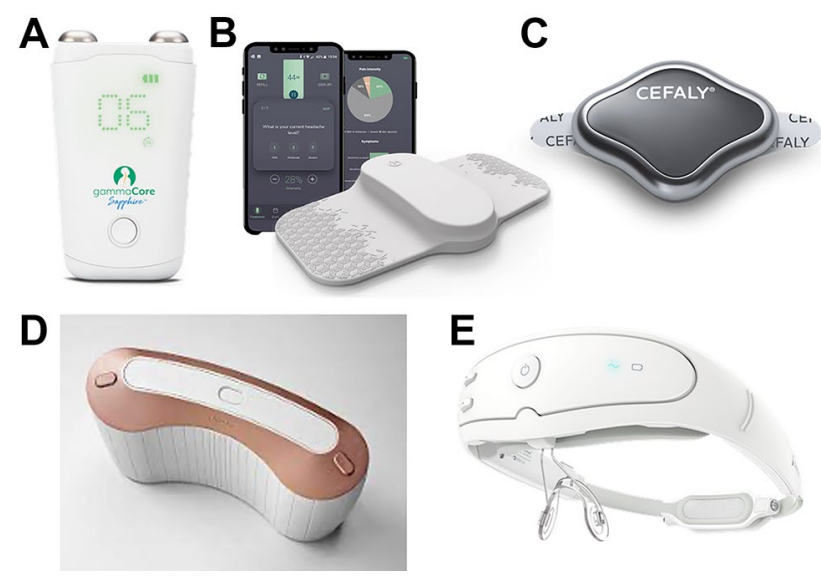

Fig. 1 A gammaCore Sapphire ${ }^{\mathrm{TM}}$, B Nerivio ${ }^{\circledR}, \mathbf{C}$ Cefaly ${ }^{\circledR}$, D sTMS mini ${ }^{\mathrm{TM}}, \mathbf{E}$ Relivion ${ }^{\circledR}$. Images were adapted from the companies' websites

sham-controlled trial for CM prevention showed no statistical significance for efficacy endpoints (monthly headache days change after 2 months; -1.4 vs. $-0.2, p=0.59$ ). However, results suggested that more extended use may be beneficial, as 15 completers had a mean change of $-7.9(95 \% \mathrm{CI}-11.9$ to $-3.8, p<0.01$ ) after 8 months of treatment [2]. The PREMIUM II study was a randomized sham-controlled doubleblind trial that enrolled CM and EM patients (8-20 headache days per month) but was terminated early due to the COVID19 pandemic. It enrolled 300 subjects and studied a predetermined modified intention-to-treat (mITT) group of 113 subjects. Based on a recent press release, PREMIUM II found a non-significant decrease in monthly migraine days (verum vs. sham: -3.1 vs. -2.3 headache days, $p=0.233$ ) in the mITT group [3]. Within the mITT group, $44.9 \%$ of verumtreated subjects had at least a $50 \%$ decrease in the number of migraine days compared to $26.8 \%$ of sham $(p=0.048)$. The effect on CM, however, was not specifically described. The gammaCore Sapphire ${ }^{\mathrm{TM}}$ device is safe and well-tolerated without any significant treatment-related adverse effects. Facial pain, gastrointestinal symptoms, and upper respiratory tract infection are the most common adverse effects in the trials for CM. Overall, nVNS may be useful for CM, but more studies are needed.

\section{Remote Electronic Neuromodulation}

Nerivio® (Theranica Bio-Electronics Ltd., Montclair, NJ) is a remote electronic neuromodulation (REN) device cleared by the FDA for use in the acute treatment of migraine (EM and $\mathrm{CM}$ ) patients 12 years and older. It consists of an armband that emits electric stimuli controlled by a smartphone app. Patients are recommended to start within $60 \mathrm{~min}$ of migraine or aura onset. The stimulation lasts for $45 \mathrm{~min}$ at an intensity level that the individual user may change via the smartphone app, which also has a migraine diary that can log headaches and session usage.

After two randomized double-blind sham-controlled trials that led to the initial FDA clearance of Nerivio for use in EM in 2019 [4, 5], two open-label observational studies (TCH-005, TCH-006) led to the FDA clearance for CM in 2020. TCH-005 study enrolled 42 subjects, and a total of 210 evaluable treatments were conducted from 38 participants [6]. Pain relief and pain freedom at $2 \mathrm{~h}$ in $\geq 50 \%$ of treated attacks were achieved by $73.7 \%$ (95\%CI 60.0-87.4) and $26.3 \%$ (95\%CI 13.4-43.1) participants, respectively. Sustained pain relief and pain freedom at $24 \mathrm{~h}$ in $\geq 50 \%$ of treated attacks were achieved by $84.4 \%$ (27/32; 95\%CI 71.8-97.0) and $45.0 \%$ (9/20; 95\% CI 23.2-66.8). In the TCH-006 study, a total of 493 evaluable treatments from 91 participants (126 enrolled) were evaluated [7]. Pain relief and pain freedom at $2 \mathrm{~h}$ were achieved by $59.3 \%$ (95CI\% 48.5-69.5) and $20.9 \%$ (95\% CI 13.0-30.6) of the participants, respectively. $57.1 \%$ (95\%CI 46.3-67.4) of the participants experienced pain relief in $\geq 50 \%$ of treated attacks. Device-related adverse events were mostly related to topical peripheral sensations of warmth, itching, arm pain, redness, and numbness.

\section{Electrical Trigeminal Nerve Stimulation}

The electrical trigeminal nerve stimulation (eTNS) device developed by CEFALY-Technology (Seraing, Belgium) was cleared by the FDA for acute and preventative treatment of migraine in adult patients. In late 2020, CEFALY $®$ DUAL (the next-generation Cefaly device) received over-the-counter clearance from the FDA. The CEFALY DUAL is programmable for ACUTE or PREVENTIVE settings. The ACUTE program is a 60-min session for acute relief at a high frequency $(100 \mathrm{~Hz})$. The PREVENT program is a 20 -min daily program for headache prevention at a low frequency $(60 \mathrm{~Hz})$.

The CEFALY device demonstrated its efficacy in the acute and preventive treatment of migraine in two randomized controlled trials [8, 9], but only one mentioned CM [8]. In the ACME study, which was a randomized doubleblind sham-controlled trial on patients with migraine (CM probably included but not specified), acute use of CEFALY at the onset of headache resulted in more pain reduction than did the sham group $(-3.46 \pm 2.32$ vs. $-1.78 \pm 1.89$; $p<0.001)$. The study population probably comprised mostly $\mathrm{EM}$, as patients who had been on Botox in the past 4 months were excluded [8].

There are three open-label observation studies (two CEFALY, one supraorbital TENS) designed for CM. In a multicenter study enrolling 23 patients, 4 patients dropped out, with 3 being intolerant to the device from worsening headache or neck tension, and one due to 







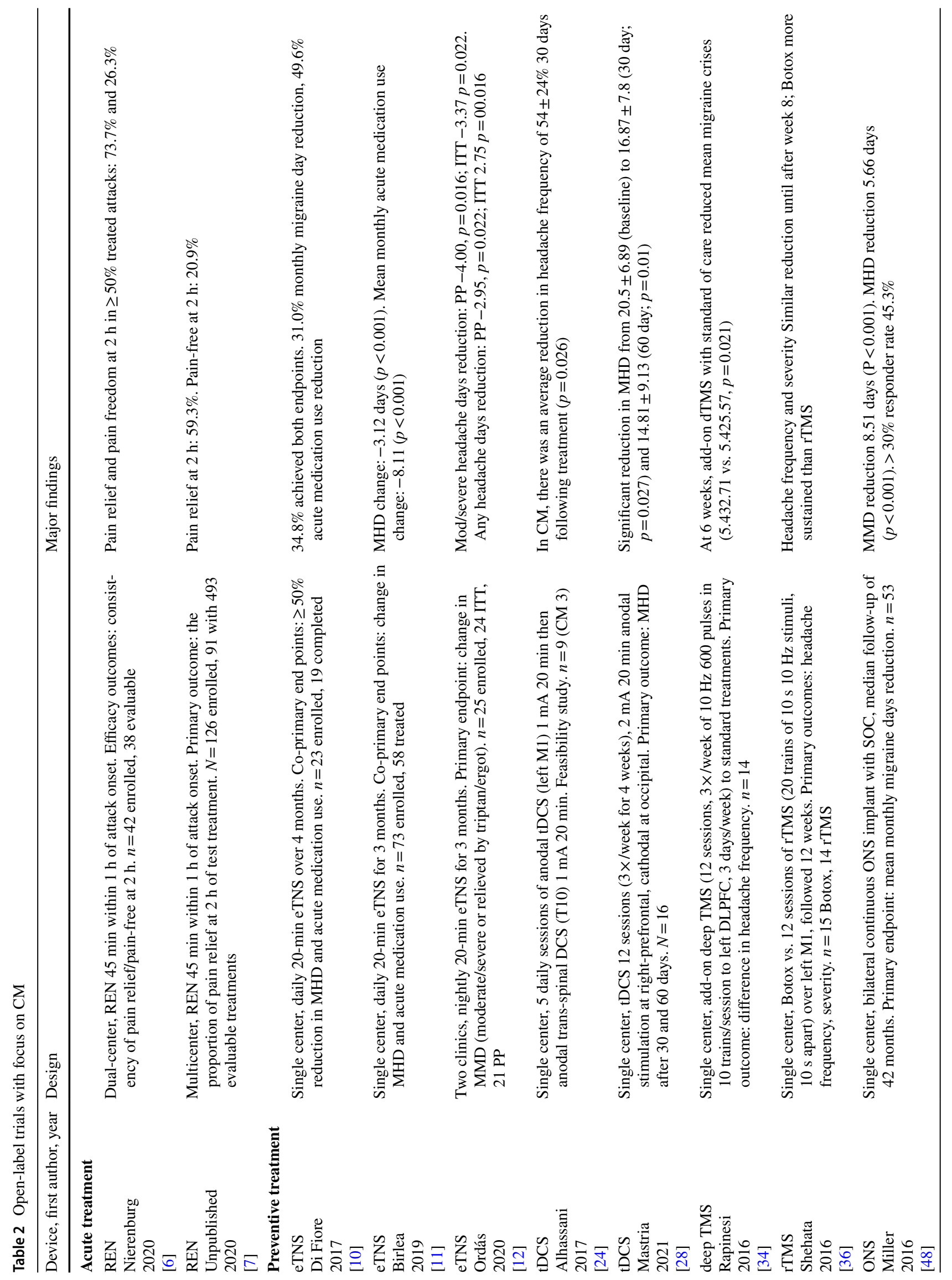




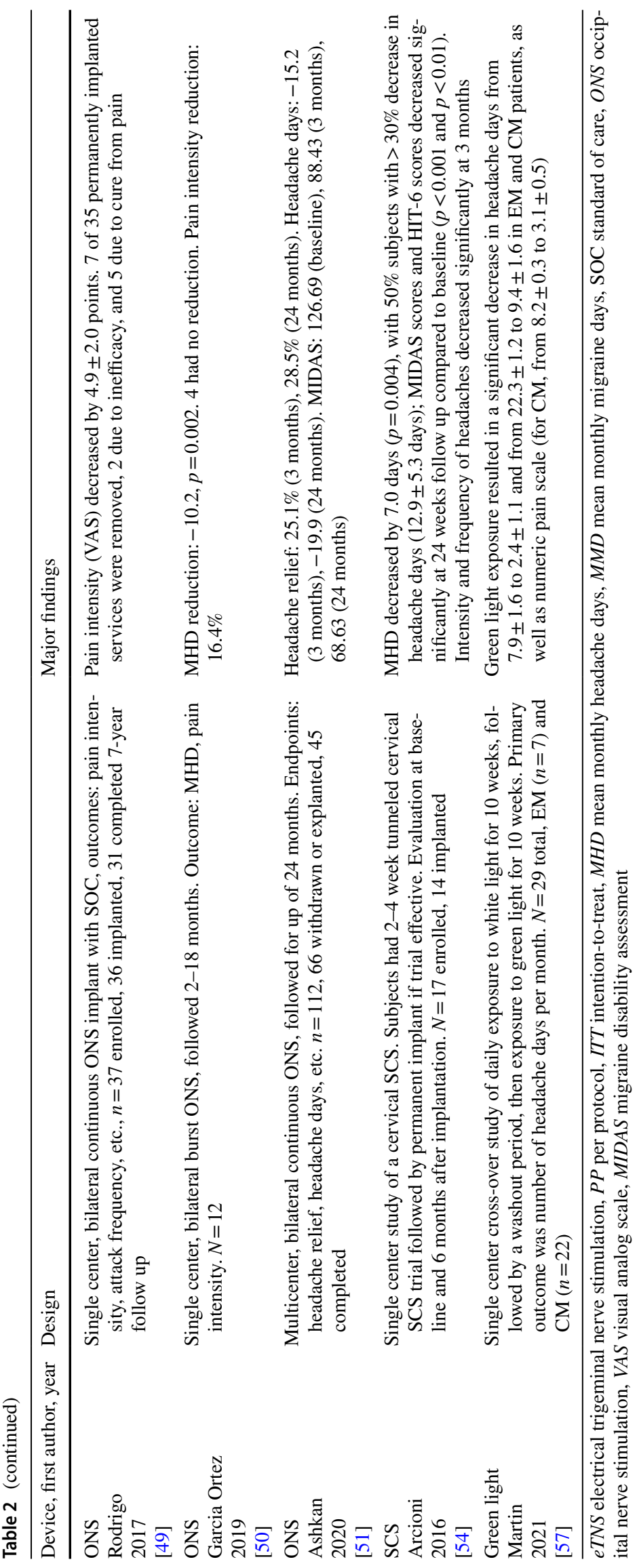


keratoconjunctivitis. For the remaining 19 patients, 34.8\% of patients achieved both endpoints. Overall, there were $31.0 \%$ decrease in monthly migraine days and $49.6 \%$ decrease in acute medication consumption after 4 months [10]. In a single-center study of 73 enrolled patients, 58 entered the treatment phase. Monthly headache days decreased from 22.55 to 19.43 days $(-16.21 \%, p<0.001)$ in the total cohort. Mean monthly acute medication intake was significantly reduced from 26.33 to $18.22(-30.81 \%$, $p<0.001)$. By the end of the study, $44.12 \%$ of patients with non-continuous headache had reversed to an EM pattern at the end of study (17.2 to 7.5 headache days) [11]. For supraorbital TENS, in a single-center study, 25 participants were recruited with 3 dropouts due to perceived lack of effectiveness and one due to lack of reliability. They were monitored over 4 months with a 1-month baseline followed by 3 more monthly visits with active treatment. The per-protocol (PP) analysis included 21 subjects, while intention-to-treat (ITT) analysis included 24 patients with the exclusion of one patient. In the PP analysis, there was a mean reduction in headache days $(-2.43$ days, $p=0.05)$ and moderate to severe headache $(-1.29$ days, $p=0.06)$, while in ITT analysis, there was a non-significant mean reduction in headache days $(-1.92$ days, $p=0.08)$ and moderate to severe headache days $(-1.20$ days, $p=0.05)$ [12]. Among 2000 patients surveyed, there was less than $5 \%$ reporting of adverse events, all minor and fully reversible. The most common adverse events reported were paresthesia (2.03\%), arousal changes (most commonly fatigue, sometimes insomnia, $0.82 \%)$, headache $(0.52 \%)$, and local skin allergy to the electrode $(0.09 \%)$ [13]. The results from these open-label studies indicate that eTNS may be useful in $\mathrm{CM}$.

\section{Single-Pulse Transcranial Magnetic Stimulation}

The sTMS mini ${ }^{\mathrm{TM}}$, which is produced by eNeura Inc. (Baltimore, MD), delivers a single-pulse (intensity $0.9 \mathrm{~T}$, rise time $180 \mu \mathrm{s})$ stimulation to the back of the head. It likely alters the cerebral cortex excitability by blocking cortical spreading depolarization waves and inhibiting thalamocortical signaling [14]. The device was initially designed to target patients with EM with aura. It is now FDA-cleared for acute and prophylactic treatment of migraine in patients 12 years of age and older. There are preventive and acute treatment protocols. The preventive protocol entails twice-daily treatment with 4 pulses ( 2 consecutive pulses, wait $15 \mathrm{~min}$, then repeat another 2 consecutive pulses). If needed for acute treatment, 3 sequential pulses can be given at the onset of the migraine attack, followed by additional pulses at 15-min intervals if needed.

Its initial FDA clearance came after a randomized doubleblind sham-controlled study [15]. There was one open-label observational study that included CM in the past 5 years.
The ESPOUSE study included 13 (10\%) CM patients who applied daily preventive use (4 pulses twice daily) and acute use ( 3 pulses, may repeat every 15 min for 2 more sessions) [16]. The effect of daily sTMS use on CM was not specifically described. The study showed that sTMS appeared efficacious in decreasing monthly headache days, acute medication use, and Headache Impact Test-6 (HIT-6) score. sTMS treatment is well tolerated, with the most reported adverse effects of lightheadedness (3.7\%), tingling (3.2\%), and tinnitus $(3.2 \%)$ [16].

\section{Combined Occipital and Trigeminal Nerve Stimulation}

Relivion ${ }^{\circledR}$ is a self-administered stimulation device that delivers electrical pulses to six branches of both the occipital and trigeminal nerves. It is pre-loaded to deliver 6 cycles of treatment (unlimited stimulation for $48 \mathrm{~h}$ ) as needed. The FDA in early 2021 cleared its use for acute treatment of migraine upon a multi-center study (RIME study) of 131 EM patients applying $1 \mathrm{~h}$ of stimulation acutely [17]. In an earlier randomized double-blind sham-controlled study of 55 migraine subjects (CM unspecified), pain intensity (measured by visual analog scale) decreased more in the treatment group at all time points (group difference at $1 \mathrm{~h} 41 \%$, $p=0.0002 ; 2$ h $33 \%, p=0.03 ; 24$ h $36 \%, p=0.02) . \geq 50 \%$ responder rates were also higher in the treatment than in the sham group at $1 \mathrm{~h}(67 \%$ ver. $20 \%, p=0.001), 2 \mathrm{~h}(67 \%$ ver. $32 \%, p=0.02)$, and $24 \mathrm{~h}(78 \%$ ver. $48 \%, p=0.04)$ [18]. No serious adverse event was noted. It is worth noting that in a retrospective study using combined occipital and trigeminal stimulation via an implanted device for refractory CM, 75\% (4/16) and 50\% (8/16) demonstrated short-term and longterm benefits respectively [19]. Relivion may thus work for $\mathrm{CM}$, but the data remains very limited.

\section{Investigational Devices}

\section{Transcranial Direct Current Stimulation}

Transcranial direct current stimulation (tDCS), which is a device utilizing a weak current (1-2 mA) delivered via sponge electrodes of various montages (anode and cathode positions), has been studied for headache disorders. Although the exact mechanism is unclear, it may affect network-level neural information coding with no direct impact on neural spiking or membrane potential $[20,21]$. It may modulate brain connectivity, thereby boosting placebo and blunting nocebo effects [22]. It is worth noting that the majority of the tDCS migraine trials were pilot studies of low to moderate quality [23]. Significant variations exist in stimulation duration, current ampere, polarity, montage, and 
the number of sessions; there remains no optimized stimulation protocol.

In the past 5 years, there were four CM-focused tDCS randomized sham-controlled trials (two probably singleblind) and two open-label studies but of significantly dissimilar study design [24-29]. Andrade et al. showed anodal but not sham stimulation of left primary motor (M1) or dorsolateral prefrontal cortex (DLPFC) reduced HIT-6 score and pain intensity [25]. Dalla Volta et al. reported cathodal stimulation of the coolest point in the forehead resulted in greater reduction than sham stimulation in monthly headache days, attack frequency, and attack duration [26]. Two open-label small studies also reported reductions in headache frequency 30 days after anodal stimulation [26, 28]. However, Cerrahoglu Sirin et al. found no difference in monthly headache days 1 month after anodal or sham stimulation [29]. A larger study by Grazzi et al. also reported no difference among anodal tDCS, cathodal tDCS, and sham at 6 and 12 months on CM patients getting acute withdrawal treatment from medication overuse [27]. In short, it remains uncertain whether $\mathrm{tDCS}$ is a useful preventive for CM, especially months after the stimulation. A standardized protocol (e.g., polarity, montage, session number, and repeat interval) and endpoints should be implemented for future tDCS trials.

\section{Repetitive Transcranial Magnetic Stimulation}

Repetitive transcranial magnetic stimulation (rTMS) devices deliver multiple brief pulses to produce a small focal cortical electrical current at the desired cortical region, such as M1 and DLPFC, affecting motor-thalamus-brainstem and prefrontal-thalamic-cingulate signaling pathways, respectively. Typically, high-frequency $(>5-20 \mathrm{~Hz})$ stimulations likely enhance cortical excitability, influence various neurotransmitter/opioidergic networks, and modulate neuronal plasticity [30-32]. The FDA has cleared it for use in major depression and obsessive-compulsive disorders. Although demonstrating a good safety profile, rTMS remains investigational for pain disorder, post-traumatic headache, and primary headache disorders. Based on two recent systematic reviews, high-frequency rTMS over the motor cortex demonstrated efficacy as a migraine treatment, but further high-quality RCTs with a standardized protocol are required to validate a treatment effect $[23,33]$.

In the past 5 years, there were 2 open-label trials and 3 randomized-controlled trials studying rTMS for use in CM [34-38]. Three open-label studies included CM but reported no efficacy data on CM [39-41] thus not discussed here. In an open-label study, Rapinesi et al. reported deep TMS (H1 coil to left DLPFC, ten $10 \mathrm{~Hz}$ trains per session, 3 weekly sessions in alternating days for 4 weeks) add-on to standard treatment and found a significant reduction in migraine frequency, rescue medication use, pain intensity, and depression rating score after 4 and 6 weeks [34]. When compared to onabotulinumtoxin A, Shehata et al. showed that rTMS (20 trains of $10010 \mathrm{~Hz}$ stimuli over left M1 3 days/week for 1 month) is of comparable efficacy to onatobulinumtoxin-A injection in CM therapy, but with less sustained effect after 8 weeks [36]. Kalita et al. compared left M1 rTMS ( 3 true sessions vs. 1 true session and 2 sham sessions; single-blind) for 3 months on patients with $\mathrm{CM}$ and chronic tension-type headache (all patients were advised to stop preventive medications 15 days prior to the randomization). There were within-group but no between-group difference on headache frequency after rTMS in 3 months. In a subgroup analysis of $82 \mathrm{CM}$ patients, there was a significant between-group difference on migraine frequency. The $\geq 50 \%$ reduction in monthly headache frequency at 2 months was significantly on intention-to-treat analysis ( 62.5 vs. $35.3 \%$; $P=0.01$ ); data from other time points were not reported [35]. In a study of rTMS for CM with medication overuse headache (MOH), Granato et al. found no benefit of rTMS (10 trains of $4020 \mathrm{~Hz}$ stimuli over left DLPFC, 5-day sessions/week for 2 weeks) over sham stimulation. There was no difference in the number of monthly headache days, symptomatic drug, and MIDAS after 120 days; the author stated rTMS has a high potential for inducing a placebo effect [37]. It is worth noting that this study utilized a sham stimulator able to induce the same skin vibratory sensation; whether such vibratory stimulation produces an active effect is unknown. In another study, Kumar et al. utilized fMRIguided neuro-navigation for accurate localization of the M1 and maintained the location accuracy in multiple sessions. They showed that in CM patients, 10 sessions of rTMS (600 pulses in 10 trains at $10 \mathrm{~Hz}$ over left M1) reduced headache intensity, frequency, and MIDAS after treatment and at 3-month follow-up, while a sham stimulation (coil placed perpendicular to skull) did not [38]. No between-group analysis was done. Overall, there were more positive results from stimulating rTMS on M1 than DLPFC [32]. rTMS of M1 may be a potential preventive strategy for CM. However, their study designs varied significantly. A standardized protocol (e.g., stimulation site/frequency and number of pulses/ sessions) and endpoints should be implemented for future tDCS trials.

\section{Occipital Nerve Stimulation}

Occipital nerve stimulation (ONS) devices, with implantable electrodes fixated near the occipital nerves and a pulse generator in the chest, have been studied for occipital neuralgia and refractory $\mathrm{CM}$ for many years. ONS may normalize the loss of condition pain modulation or directly counteract the trigeminally mediated central sensitization in these patients $[42,43]$. Several multi-center randomized sham-controlled trials demonstrated improvement in headache frequency, 
intensity, or disability [44-47]; all were published more than 5 years ago.

In the past 5 years, there were 4 open-label studies on CM published [48-51]. Most studies reported continuous ONS except one utilized burst ONS [50], which elicited subthreshold sensory perception and still seemed to reduce pain intensity and headache days. The stimulation parameters and study endpoints, however, varied considerably between studies. Miller et al. analyzed a cohort of 53 intractable CM patients (media follow-up 42 months after bilateral ONS electrodes implantation) and found an 8.51-day reduction $(p<0.001)$ in monthly moderate-to-severe headache days [48]. Similarly, Rodrigo et al. studied 37 refractory CM patients (average follow-up $9.4 \pm 6.1$ years) and showed substantial pain reduction (VAS decreased by $4.9 \pm 2.0$ points). Five were pain-free at their last visit. Seven required explantation, with 2 due to lack of efficacy and 5 due to complete pain resolution [49]. Using burst ONS, Garcia-Ortega et al. also showed a significant reduction of 10.2 monthly headache days ( $p=0.002$, one-tailed) in $12 \mathrm{CM}$ patients [50]. The recent multi-center, international open-label RELIEF study recruited 132 intractable CM patients (45 completed 24-month visit) implanted with Abbott ONS and demonstrated headache pain relief, decrease in headache days, and headache disability [51]. The spatial sensory field and quality of the ONS seemed correlated with the clinical effectiveness reported by the patient [52]. However, there were adverse events of infection, lead migration, and stimulationrelated symptoms in up to $20 \%$ of patients after 1 year [51]. Due to these technical issues, new leads with anchors are being developed and will hopefully circumvent the adverse event profile of currently available systems. ONS seems a promising device for $\mathrm{CM}$. Unfortunately, no ONS device has yet received FDA clearance for use in migraine.

\section{Spinal Cord Stimulation}

Spinal cord stimulation (SCS), which has been used over the past three decades for chronic pain of neuropathic origin, has been applied to patients with intractable headache in several small case series. The SCS electrodes are threaded into high-cervical epidural space $(\mathrm{C} 2 / 3)$ with a pulse generator implanted subcutaneously in the paraumbilical or gluteal position. High-cervical SCS for intractable CM was first reported by De Agostino et al. in a small case series. After a median of 15 months (range 2-48) since implantation, mean pain intensity was significantly reduced by $60 \%(p<0.0001)$, with $71 \%$ of the patients experiencing a decrease of $50 \%$ or more. A reduction in the median number of migraine days and medication use along with improved quality of life was also observed. However, 3 had infections and 3 had lead dislocations [53]. In a prospective open-label study using highfrequency $10 \mathrm{kHz}$ cervical SCS on CM patients refractory to onabotulinumtoxin-A, 17 underwent SCS trial, and 14 received SCS implantation. After 6 months, seven of the 14 subjects had a $>30 \%$ reduction in headache days. However, 3 subjects reported tenderness over pulse generator/connection site, and one had lead migration requiring surgical revision [54]. The efficacy of SCS on CM remains uncertain.

\section{Green Light}

Exposure to green light may modulate nociception and anxiety. Non-green light stimuli exacerbated pain intensity during the migraine attack but not in healthy control [55]. In contrast, exposure to green light reduced pain intensity in $\sim 20 \%$ of the patients (18/69 CM) [56]. In a small crossover study (4-week washout), comparing 1-2 $\mathrm{h}$ of daily white light and green light for ten weeks in 29 migraine subjects (22 CM), Martin et al. found that green light but not white light exposure resulted in a significant reduction in the number of headache in CM patients $(22.3 \pm 1.2$ to $9.4 \pm 1.6$, $p<0.001)$. Green light exposure also reduced headache impact (HIT-6) and improved quality of life (EQ-5D-5L). There were no adverse effects reported. Despite having a small case number in this study, the superior efficacy and safety profile may warrant further investigation [57].

\section{Clinical Perspective}

There are now nearly half a dozen FDA-cleared devices for migraine, and several prior clearances have now been expanded to include adolescent patients aged 12 or older. It is important to know that not all FDA-cleared devices were properly studied for CM. The only randomized shamcontrolled trial for CM was by Silberstein et al. using gammaCore device. Unfortunately, the study did not meet its efficacy endpoint. Several open-label observational studies utilized CEFALY or Nerivio to evaluate pain reduction in patients with CM. These studies are not blinded and suffer from selection and reporting biases. Some studies included both EM and CM patients but reported no description of the case number of $\mathrm{CM}$ nor response relevant to $\mathrm{CM}$. In addition, due to the lack of trial guidelines that recognized the unique approach and challenge of a neuromodulation device trial for migraine, there exists a wide variation in endpoints, types of control, and study population for analysis (intentionto-treat vs. per-protocol), making study comparison difficult. The International Headache Society has therefore published recommendations for assessing neuromodulation devices in the acute and preventive treatmentof migraine [58••]. As the use of these devices becomes widespread, we anticipate more well-powered and high-quality studies adhering to clinical trial guidelines to fully determine the benefit of these devices in CM. More high-quality trials hopefully will 
also facilitate the insurance companies to expand the coverage to more neuromodulation devices.

\section{Conclusion}

Neuromodulation devices are emerging therapy complementing the traditional pharmacotherapies for migraine. Noninvasive neuromodulation is generally considered safe with minimal adverse events, but certain safety precautions should still be noted. To date, there are ongoing investigations into the utility of FDA-cleared or investigational devices for CM patients. With increasing ease of use and the ability to customize stimulation strength, these devices encapsulate the personalization of medicine. Improving the quality of such trials will enhance these devices' clinical recognition and hopefully expand future insurance coverage. The guideline from the International Headache Society for migraine clinical trials with neuromodulation devices will aid significantly in this effort. The development of safe and effective treatments for CM is essential for providing patients with the most optimized care.

\section{Compliance with Ethical Standards}

Conflict of Interest Yuan Hsiangkuo reports receiving consultant fees from Trillen Medical. Tzu-Ying Chuang declares no conflict of interest.

Human and Animal Rights and Informed Consent This article does not contain any studies with human or animal subjects performed by any of the authors.

\section{References}

Papers of particular interest, published recently, have been highlighted as:

\section{$\bullet$ Of major importance}

1. GBD 2017 Disease and Injury Incidence and Prevalence Collaborators. Global, regional, and national incidence, prevalence, and years lived with disability for 354 diseases and injuries for 195 countries and territories, 1990-2017: a systematic analysis for the Global Burden of Disease Study 2017. Lancet. 2018;392(10159):1789-858. https://doi.org/10.1016/ S0140-6736(18)32279-7.

2. Silberstein SD, Calhoun AH, Lipton RB, Grosberg BM, Cady $\mathrm{RK}$, Dorlas S, et al. Chronic migraine headache prevention with noninvasive vagus nerve stimulation: the EVENT study. Neurology. 2016;87(5):529-38. https://doi.org/10.1212/WNL. 0000000000002918 .

3. electroCore announces positive top-line results from PREMIUM II migraine prevention study. https://www.globenewswire.com/newsrelease/2020/12/08/2141280/0/en/electroCore-Announces-PositiveTop-Line-Results-from-PREMIUM-II-Migraine-Prevention-Study. html. (2020). Accessed Mar. 32021.
4. Yarnitsky D, Dodick DW, Grosberg BM, Burstein R, Ironi A, Harris D, et al. Remote electrical neuromodulation (REN) relieves acute migraine: a randomized, double-blind, placebocontrolled, multicenter trial. Headache. 2019;59(8):1240-52. https://doi.org/10.1111/head.13551.

5. Yarnitsky D, Volokh L, Ironi A, Weller B, Shor M, Shifrin A, et al. Nonpainful remote electrical stimulation alleviates episodic migraine pain. Neurology. 2017;88(13):1250-5. https://doi.org/ 10.1212/WNL.0000000000003760.

6. Nierenburg H, Vieira JR, Lev N, Lin T, Harris D, Vizel M, et al. Remote electrical neuromodulation for the acute treatment of migraine in patients with chronic migraine: an open-label pilot study. Pain Ther. 2020;9(2):531-43. https://doi.org/10.1007/ s40122-020-00185-1.

7. Pilot study with chronic migraine patients. https://theranica.com/ technology/. Accessed 5/20 2021.

8. Chou DE, Shnayderman Yugrakh M, Winegarner D, Rowe V, Kuruvilla D, Schoenen J. Acute migraine therapy with external trigeminal neurostimulation (ACME): a randomized controlled trial. Cephalalgia. 2019;39(1):3-14. https://doi.org/10.1177/ 0333102418811573.

9. Schoenen J, Vandersmissen B, Jeangette S, Herroelen L, Vandenheede M, Gerard P, et al. Migraine prevention with a supraorbital transcutaneous stimulator: a randomized controlled trial. Neurology. 2013;80(8):697704. https://doi.org/10.1212/WNL.0b013e3182825055.

10. Di Fiore P, Bussone G, Galli A, Didier H, Peccarisi C, D'Amico $\mathrm{D}$, et al. Transcutaneous supraorbital neurostimulation for the prevention of chronic migraine: a prospective, open-label preliminary trial. Neurol Sci. 2017;38(Suppl 1):201-6. https://doi. org/10.1007/s10072-017-2916-7.

11. Birlea M, Penning S, Callahan K, Schoenen J. Efficacy and safety of external trigeminal neurostimulation in the prevention of chronic migraine: an open-label trial. Cephalalgia Reports. 2019;2:1-10. https://doi.org/10.1177/2515816319856625.

12. Ordás CM, Cuadrado ML, Pareja JA, de-las-Casas-Cámara G, Gómez-Vicente L, Torres-Gaona G, et al. Transcutaneous supraorbital stimulation as a preventive treatment for chronic migraine: a prospective, open-label study. Pain Medicine. 2020;21(2):415-22.

13. Magis D, Sava S, d'Elia TS, Baschi R, Schoenen J. Safety and patients' satisfaction of transcutaneous supraorbital neurostimulation (tSNS) with the Cefaly(R) device in headache treatment: a survey of 2,313 headache sufferers in the general population. J Headache Pain. 2013;14(1):95. https://doi.org/10.1186/ 1129-2377-14-95.

14. Barker AT, Shields K. Transcranial magnetic stimulation: basic principles and clinical applications in migraine. Headache. 2017;57(3):517-24. https://doi.org/10.1111/head.13002.

15. Lipton RB, Dodick DW, Silberstein SD, Saper JR, Aurora SK, Pearlman SH, et al. Single-pulse transcranial magnetic stimulation for acute treatment of migraine with aura: a randomised, double-blind, parallel-group, sham-controlled trial. Lancet Neurol. 2010;9(4):373-80. https://doi.org/10.1016/S1474-4422(10) 70054-5.

16. Starling AJ, Tepper SJ, Marmura MJ, Shamim EA, Robbins MS, Hindiyeh N, et al. A multicenter, prospective, single arm, open label, observational study of sTMS for migraine prevention (ESPOUSE Study). Cephalalgia. 2018;38(6):1038-48. https:// doi.org/10.1177/0333102418762525.

17. Relivion ${ }^{\circledR}$ wearable brain neuromodulation technology cleared by FDA for the treatment of migraine. https://www.businesswire.com/ news/home/20210302005356/en/Relivion\%C2\%AE-Wearable-BrainNeuromodulation-Technology-Cleared-by-FDA-for-the-Treatment-ofMigraine. (2021). Accessed 03/02 2021.

18. Daniel O, Tepper SJ. First non-invasive combined occipital $\&$ trigeminal nerve stimulation digital therapeutics system for 
treatment of migraine: a randomized, sham-controlled, doubleblind clinical trial. 61st Annual Scientific Meeting American Headache Society. Philadelphia2019.

19. Clark SW, Wu C, Boorman DW, Chalouhi N, Zanaty M, Oshinsky $\mathrm{M}$, et al. Long-term pain reduction does not imply improved functional outcome in patients treated with combined supraorbital and occipital nerve stimulation for chronic migraine. Neuromodulation. 2016;19(5):507-14. https://doi.org/10.1111/ner.12400.

20. Liu A, Voroslakos M, Kronberg G, Henin S, Krause MR, Huang $\mathrm{Y}$, et al. Immediate neurophysiological effects of transcranial electrical stimulation. Nat Commun. 2018;9(1):5092. https://doi. org/10.1038/s41467-018-07233-7.

21. Voroslakos M, Takeuchi Y, Brinyiczki K, Zombori T, Oliva A, Fernandez-Ruiz A, et al. Direct effects of transcranial electric stimulation on brain circuits in rats and humans. Nat Commun. 2018;9(1):483. https://doi.org/10.1038/s41467-018-02928-3.

22. Tu Y, Wilson G, Camprodon J, Dougherty DD, Vangel M, Benedetti F, et al. Manipulating placebo analgesia and nocebo hyperalgesia by changing brain excitability. Proc Natl Acad Sci U S A. 2021;118(19): e2101273118. https://doi.org/10.1073/ pnas. 2101273118

23. Stilling JM, Monchi O, Amoozegar F, Debert CT. Transcranial magnetic and direct current stimulation (TMS/tDCS) for the treatment of headache: a systematic review. Headache. 2019;59(3):33957. https://doi.org/10.1111/head.13479.

24. Alhassani G, Treleaven J, Schabrun SSM. Combined transcranial and trans-spinal direct current stimulation in chronic headache: a feasibility and safety trial for a novel intervention. Hong Kong Physiother J. 2017;37:1-9. https://doi.org/10.1016/j.hkpj.2016.11.001.

25. Andrade SM, de Brito Aranha REL, de Oliveira EA, de Mendonca C, Martins WKN, Alves NT, et al. Transcranial direct current stimulation over the primary motor vs prefrontal cortex in refractory chronic migraine: a pilot randomized controlled trial. J Neurol Sci. 2017;378:225-32. https://doi.org/10.1016/j. jns.2017.05.007.

26. Dalla Volta G, Marceglia S, Zavarise P, Antonaci F. Cathodal tDCS guided by thermography as adjunctive therapy in chronic migraine patients: a Sham-controlled pilot study. Front Neurol. 2020;11(121):121. https://doi.org/10.3389/fneur.2020.00121.

27. Grazzi L, Usai S, Bolognini N, Grignani E, Sansone E, Tramacere I, et al. No efficacy of transcranial direct current stimulation on chronic migraine with medication overuse: a double blind, randomised clinical trial. Cephalalgia. 2020;40(11):1202-11. https:// doi.org/10.1177/0333102420931050.

28. Mastria G, Vigano A, Corrado A, Mancini V, Pirillo C, Badini $\mathrm{S}$, et al. Chronic migraine preventive treatment by prefrontaloccipital transcranial direct current stimulation (tDCS): a proof-of-concept study on the effect of psychiatric comorbidities. Front Neurol. 2021;12(592): 654900. https://doi.org/10. 3389/fneur.2021.654900.

29. Cerrahoglu Sirin T, Aksu S, Hasirci Bayir BR, Ulukan C, Karamursel S, Kurt A, et al. Is allodynia a determinant factor in the effectiveness of transcranial direct current stimulation in the prophylaxis of migraine? Neuromodulation. 2021;24(5):899-909. https://doi.org/10.1111/ner.13409.

30. Henssen D, Derks B, van Doorn M, Verhoogt N, Van Cappellen van Walsum AM, Staats $P$, et al. Vagus nerve stimulation for primary headache disorders: an anatomical review to explain a clinical phenomenon. Cephalalgia. 2019;39(9):1180-94. doi: https://doi.org/10.1177/0333102419833076.

31. Iglesias AH. Transcranial magnetic stimulation as treatment in multiple neurologic conditions. Curr Neurol Neurosci Rep. 2020;20(1):1. https://doi.org/10.1007/s11910-020-1021-0.

32. Leung A, Shirvalkar P, Chen R, Kuluva J, Vaninetti M, Bermudes $\mathrm{R}$, et al. Transcranial magnetic stimulation for pain, headache, and comorbid depression: INS-NANS expert consensus panel review and recommendation. Neuromodulation. 2020;23(3):267-90. https://doi.org/10.1111/ner.13094.

33. Hulla R, Liegey-Dougall A. A systematic review of highfrequency transcranial magnetic stimulation on motor cortex areas as a migraine preventive treatment. Cephalalgia Reports. 2019;2:251581631988997. https://doi.org/10.1177/ 2515816319889971.

34. Rapinesi C, Del Casale A, Scatena P, Kotzalidis GD, Di Pietro $S$, Ferri VR, et al. Add-on deep transcranial magnetic stimulation (dTMS) for the treatment of chronic migraine: a preliminary study. Neurosci Lett. 2016;623:7-12. https://doi.org/10. 1016/j.neulet.2016.04.058.

35. Kalita J, Laskar S, Bhoi SK, Misra UK. Efficacy of single versus three sessions of high rate repetitive transcranial magnetic stimulation in chronic migraine and tension-type headache. J Neurol. 2016;263(11):2238-46. https://doi.org/10.1007/ s00415-016-8257-2.

36. Shehata HS, Esmail EH, Abdelalim A, El-Jaafary S, Elmazny A, Sabbah A, et al. Repetitive transcranial magnetic stimulation versus botulinum toxin injection in chronic migraine prophylaxis: a pilot randomized trial. J Pain Res. 2016;9:7717. https://doi.org/10.2147/JPR.S116671.

37. Granato A, Fantini J, Monti F, Furlanis G, Musho Ilbeh S, Semenic M, et al. Dramatic placebo effect of high frequency repetitive TMS in treatment of chronic migraine and medication overuse headache. J Clin Neurosci. 2019;60:96-100. https://doi.org/10.1016/j.jocn.2018.09.021.

38. Kumar A, Mattoo B, Bhatia R, Kumaran S, Bhatia R. Neuronavigation based 10 sessions of repetitive transcranial magnetic stimulation therapy in chronic migraine: an exploratory study. Neurol Sci. 2021;42(1):131-9. https://doi.org/10.1007/ s10072-020-04505-3.

39. Tripathi GM, Kalita J, Misra UK. Role of glutamate and its receptors in migraine with reference to amitriptyline and transcranial magnetic stimulation therapy. Brain Res. 2018;1696:317. https://doi.org/10.1016/j.brainres.2018.05.046.

40. Zardouz S, Shi L, Leung A. A feasible repetitive transcranial magnetic stimulation clinical protocol in migraine prevention. SAGE Open Med Case Rep. 2016;4(0):2050313X16675257. doi: https://doi.org/10.1177/2050313X16675257.

41. Chen PR, Lai KL, Fuh JL, Chen SP, Wang PN, Liao KK, et al. Efficacy of continuous theta burst stimulation of the primary motor cortex in reducing migraine frequency: a preliminary open-label study. J Chin Med Assoc. 2016;79(6):304-8. https:// doi.org/10.1016/j.jcma.2015.10.008.

42. Wodehouse T, Bahra A, Mehta V. Changes in peripheral and central sensitization in patients undergoing occipital nerve stimulation. Br J Pain. 2020;14(4):250-5. https://doi.org/10.1177/ 2049463719860548.

43. Gobel CH, Heinze A, Karstedt S, Clasen S, Gobel H. Effect of occipital nerve stimulation (ONS) on the orbicularis oculi reflex triggered by a standardized air flow in patients with chronic migraine-a prospective, randomized, interventional study. Pain Ther. 2021;10(1):567-76. https://doi.org/10.1007/ s40122-021-00242-3.

44. Silberstein SD, Dodick DW, Saper J, Huh B, Slavin KV, Sharan A, et al. Safety and efficacy of peripheral nerve stimulation of the occipital nerves for the management of chronic migraine: results from a randomized, multicenter, double-blinded, controlled study. Cephalalgia. 2012;32(16):1165-79. https://doi.org/ $10.1177 / 0333102412462642$.

45. Saper JR, Dodick DW, Silberstein SD, McCarville S, Sun M, Goadsby PJ, et al. Occipital nerve stimulation for the treatment of intractable chronic migraine headache: ONSTIM feasibility study. Cephalalgia. 2011;31(3):271-85. https://doi.org/10.1177/ 0333102410381142. 
46. Serra G, Marchioretto F. Occipital nerve stimulation for chronic migraine: a randomized trial. Pain Physician. 2012;15(3):245-53.

47. Dodick DW, Silberstein SD, Reed KL, Deer TR, Slavin KV, Huh B, et al. Safety and efficacy of peripheral nerve stimulation of the occipital nerves for the management of chronic migraine: longterm results from a randomized, multicenter, double-blinded, controlled study. Cephalalgia. 2015;35(4):344-58. https://doi. org/10.1177/0333102414543331.

48. Miller S, Watkins L, Matharu M. Long-term outcomes of occipital nerve stimulation for chronic migraine: a cohort of 53 patients. J Headache Pain. 2016;17(1):68. https://doi.org/10. 1186/s10194-016-0659-0.

49. Rodrigo D, Acin P, Bermejo P. Occipital nerve stimulation for refractory chronic migraine: results of a long-term prospective study. Pain Physician. 2017;20(1):E151-9.

50. Garcia-Ortega R, Edwards T, Moir L, Aziz TZ, Green AL, FitzGerald JJ. Burst occipital nerve stimulation for chronic migraine and chronic cluster headache. Neuromodulation. 2019;22(5):638-44. https://doi.org/10.1111/ner.12977.

51. Ashkan K, Sokratous G, Gobel H, Mehta V, Gendolla A, Dowson A, et al. Peripheral nerve stimulation registry for intractable migraine headache (RELIEF): a real-life perspective on the utility of occipital nerve stimulation for chronic migraine. Acta Neurochir (Wien). 2020;162(12):3201-11. https://doi.org/10.1007/s00701-020-04372-z.

52. Gobel CH, Gobel A, Niederberger U, Heinze A, Heinze-Kuhn K, Meinecke $\mathrm{C}$, et al. Occipital nerve stimulation in chronic migraine: the relationship between perceived sensory quality, perceived sensory location, and clinical efficacy-a prospective, observational, non-interventional study. Pain Ther. 2020;9(2):615-26. https:// doi.org/10.1007/s40122-020-00194-0.
53. De Agostino R, Federspiel B, Cesnulis E, Sandor PS. Highcervical spinal cord stimulation for medically intractable chronic migraine. Neuromodulation. 2015;18(4):289-96; discussion 96. https://doi.org/10.1111/ner.12236.

54. Arcioni R, Palmisani S, Mercieri M, Vano V, Tigano S, Smith T, et al. Cervical $10 \mathrm{kHz}$ spinal cord stimulation in the management of chronic, medically refractory migraine: a prospective, openlabel, exploratory study. Eur J Pain. 2016;20(1):70-8. https:// doi.org/10.1002/ejp.692.

55. Nir RR, Lee AJ, Huntington $S$, Noseda R, Bernstein CA, Fulton AB, et al. Color-selective photophobia in ictal vs interictal migraineurs and in healthy controls. Pain. 2018;159(10):2030-4. https://doi.org/ 10.1097/j.pain.0000000000001303.

56. Noseda R, Bernstein CA, Nir RR, Lee AJ, Fulton AB, Bertisch $\mathrm{SM}$, et al. Migraine photophobia originating in cone-driven retinal pathways. Brain. 2016;139(Pt 7):1971-86. https://doi.org/10. 1093/brain/aww119.

57. Martin LF, Patwardhan AM, Jain SV, Salloum MM, Freeman J, Khanna R, et al. Evaluation of green light exposure on headache frequency and quality of life in migraine patients: a preliminary one-way cross-over clinical trial. Cephalalgia. 2021;41(2):13547. https://doi.org/10.1177/0333102420956711.

58.• Tassorelli C, Diener HC, Silberstein SD, Dodick DW, Goadsby PJ, Jensen RH, et al. Guidelines of the International Headache Society for clinical trials with neuromodulation devices for the treatment of migraine. Cephalalgia. 2021;41(11-12):1135-51. https://doi.org/10.1177/03331024211010413. Guideline paper for migraine clinical trials using neuromodulation device.

Publisher's Note Springer Nature remains neutral with regard to jurisdictional claims in published maps and institutional affiliations. 